\title{
La Pedagogía en Educación Física en Chile en contextos escolares, las universidades y las políticas públicas. Una revisión Physical Education Pedagogy in Chile in school contexts, universities and public policies. A review
}

*Sebastian Sánchez González, **Cristobal Castro Barrientos, **Arturo Prat, **Antonio Castillo-Paredes *Escuela de Suboficiales del Ejército de Chile (Chile), **Universidad de Las Américas (Chile)

Resumen. La Educación Física es un conjunto de disciplinas, desde la antigüedad su utilización estaba ligada a sociedades primitivas para la supervivencia, hacia la ética, recreación, diversión, exploración, educación, prevención e instrucción militar, a nuestros días, ha sufrido influencias de Escuelas y Corrientes Pedagógicas, con la finalidad de conseguir un ser integro a través del movimiento y sus estructuras. La presente revisión tiene por objetivo describir y analizar los artículos sobre la Educación Física en el sistema escolar, educación superior y desde las políticas públicas sobre sus orientaciones y aplicaciones a nivel escolar y formación docente de pregrado en Chile. La búsqueda de las investigaciones solo en idioma español se realizó en las bases de datos SciELO, Scopus, Dialnet y Latíndex mediante las palabras claves «educación física en chile» AND «actividad física» AND «ejercicio físico» AND «juego» AND «deporte» en un rango de 20 años desde el 2001 al 2021, donde se encontraron 31 investigaciones que cumplían con los criterios de selección. La Educación Física en Chile posee distintas Escuelas y Corrientes para dar respuesta a los beneficios biológicos, psicológicos y sociales, de acuerdo con las investigaciones encontradas, se pierde el sentido de la Educación Física desde Contextos Escolares, en la Educación Superior y en las Políticas Públicas, dado a que la profesión debe dar respuesta no tan solo a requerimientos gubernamentales, sino también a movimientos sociales nacionales e internacionales.

Palabras claves: Educación Física, Juego, Deporte, Actividad Física, Ejercicio Físico.

Abstract. Physical Education is a set of disciplines, since ancient times its use was linked to primitive societies for survival,
towards ethics, recreation, fun, exploration, education, prevention, and military instruction, to our days, it has suffered
influences from Schools and Pedagogical currents, to achieve an integral being through movement and its structures. The
purpose of this review is to describe and analyze the articles on Physical Education in the school system, higher education and
from public policies on its orientations and applications at the school level and undergraduate teacher training in Chile. The
search of the investigations only in Spanish language was carried out in the SciELO, Scopus, Dialnet and Latindex databases
using the keywords «physical education in Chile» AND «physical activity» AND «exercise» AND «game» AND «sport «in a
range of 20 years from 2001 to 2021, where 31 investigations were found that met the selection criteria. Physical Education
in Chile has different Schools and Currents to respond to the biological, psychological, and social benefits, according to the
research found, the meaning of Physical Education is lost from School Contexts, in Higher Education and in Public Policies,
because the profession must respond not only to government requirements, also to national and international social movements. Keywords: Physical Education, Play, Sport, Physical Activity, Exercise.

\section{Introducción}

La Real Academia de la Lengua Española (2019) define Educación Física como «Conjunto de disciplinas y ejercicios encaminados a lograr el desarrollo y perfección corporales». De esta manera, la Educación Física ha ido desarrollando a través del tiempo nuevos conocimientos, reflexiones y creencias desde la sociedad, la cultura y el currículo escolar (Moreno, Campos y Almonacid, 2012). Además, la Alfabetización Física es un término del siglo

Fecha recepción: 17-02-21. Fecha de aceptación: 09-09-21

Antonio Castillo-Paredes

acastillop85@gmail.com
XXI que surgió por primera vez en la literatura deportiva antes de aplicarse también a la educación física. El concepto de alfabetización física cubre una variedad de condiciones para un estudiante, tales como, motivación, confianza, competencia física, conocimiento y comprensión (Ramos, López y Murillo, 2016).

En consecuencia, si bien la Educación Física se construye de diversos componentes como el juego, la actividad física, el ejercicio físico y el deporte, o cualquier otra expresión motriz, se requiere de una variedad de condiciones para desarrollarla de manera apropiada. La historia de la Educación Física nos remonta hasta los comienzos de la humanidad, en la era primitiva, época cuyo rol principal era la sobrevivencia. Luego, encon- 
tramos vestigios de la educación física en las primeras civilizaciones y épocas de la historia como China, India, Egipto, Grecia, Roma, Edad Media, Renacimiento y Edad Modernas, evidenciando una evolución en fines éticos, religiosos, educativos y de entrenamiento militar (García \& Ruiz, 2010; Sainz, 1992; Mamonde, 1995). La Educación Física es una enseñanza que ha sufrido una constante revisión y actualización, debido a la influencia de las escuelas tales como la Escuela Sueca (Sistema Analítico), Escuela Alemana (Sistema Rítmico), Escuela Francesa (Sistema Natural) y Escuela Inglesa (Sistema Deportivo) (Gonzalez, Madrera \& Salguero, 2004), y de las diversas corrientes como la biomotriz, psicomotriz, expresivo motriz y sociomotriz (Franco, 2010) y las corrientes o tendencias de la Educación Física relacionadas con el «cuerpo acrobático» (Educación físico-deportiva), «cuerpo pensante» (Educación psicomotriz) y «cuerpo comunicante» (Expresión corporal) (Zagalaz, Moreno \& Cachón, 2001).

En Chile, entre 1889 y 1930 , y debido a la alta mortandad infantil, entre otras razones, se busca instaurar la Educación Física como política pública para el bienestar físico e intelectual de los niños y niñas (BND, 2018). En este mismo sentido, el año 2012 la Educación Física en el currículo escolar cambia de nombre debido a las alarmantes cifras de enfermedades crónicas no trasmisibles reportadas en la Encuesta Nacional de Salud 2009-2010 (Celis-Morales, et al., 2015; Díaz-Martínez, et al., 2017; Petermann, et al., 2017), pasando a llamarse Educación Física y Salud. Actualmente, las carreras de Pedagogía en Educación Física en las mallas de las universidades chilenas tienen enfoques médicos, científicos, pedagógicos, deportivos, alto rendimiento, de humanidades, inclusivos, estéticos y psicológicos (Poblete, Moreno, \& Rivera, 2014).

Como se ha mencionado, la educación física es muy importante para la salud y el bienestar general de los estudiantes. A nivel nacional, y de acuerdo con a la última Encuesta Nacional de Salud realizada el año 2017, el 39,2\% de la población del país tiene sobrepeso y el 31,2\% obesidad. Asimismo, las cifras de la Encuesta de Vulnerabilidad Infantil que realiza la Junta Nacional de Auxilio Escolar y Becas (JUNAEB) y que se aplica estudiantes de Prekínder, Kínder, Primero Básico, Quinto Básico y de Primero Medio, muestran que la obesidad va en aumento, pasando de 15,9\% del año 2009 a 25,4\% al año 2020 (JUNAEB, 2020).

Debido a las preocupantes cifras de obesidad y al rol que tiene la Educación Física en estimular una vida sana a través de la práctica de Actividad Física, Ejercicio,
Juego o el Deporte, la presente revisión tiene por objetivo describir y analizar los artículos sobre la Educación Física en el sistema escolar, educación superior y desde las políticas públicas sobre sus orientaciones y aplicaciones a nivel escolar y formación docente de pregrado en Chile.

\section{Metodología}

Para la búsqueda de las investigaciones, fueron utilizados los siguientes sistemas de búsqueda de la información y bases de datos SciELO (06), Scopus (01), Dialnet (36) Latíndex (0), con las palabras claves en español («Educación Física en Chile») AND («Actividad física») OR («Ejercicio físico») OR («Juego») OR («Deporte») OR («Recreo»). Se incluyeron artículos, trabajos de revisión, tesis de pregrado, tesis de postgrado, trabajos de revisión, descriptivos, investigaciones experimentales, cuasi experimentales, ensayos clínicos publicados entre los años 2001 y 2021, la búsqueda de la información se actualizó con fecha 05 de agosto del 2021. Los criterios de inclusión, se consideraron trabajos de investigación donde se consideraron contextos escolares, universitarios, políticos y sociales en torno a la Educación Física en Chile.

\section{Identificación}

Se identificaron un total de 43 trabajos de investigación a través de los sistemas de búsqueda de información y bases de datos. Se eliminaron un total de 4 trabajos de investigación por duplicado. Luego, fueron seleccionados un total de 39 trabajos de investigación. De los cuales, solo 35 trabajos de investigación cumplían con nuestros criterios de selección, y finalmente solo 31 fueron incluidos en la revisión (Figura 1).

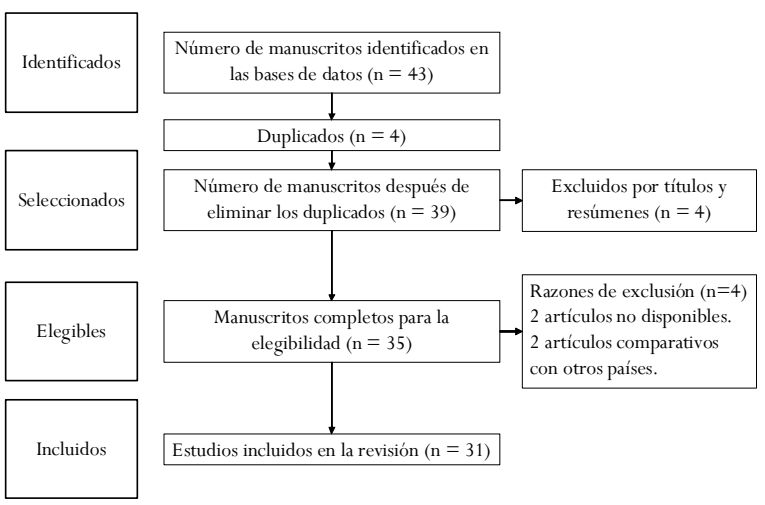

Figura 1. Identificación de estudios en la revisión.

\section{Descripción de los resultados}


Tabla 1.

Resultados de los trabajos de investigación encontrados sobre la Educación Física en Chile.

\begin{tabular}{|c|c|c|c|c|}
\hline Autores & Muestra & Documento & Objetivo & Contexto \\
\hline Acuña (2020) & $\begin{array}{l}\text { Revisión de documentos } \\
\text { ministeriales, leyes e } \\
\text { investigaciones de bases de datos. }\end{array}$ & Manuscrito & $\begin{array}{l}\text { Explorar los esfuerzos institucionales del Gobierno ibañista por legitimar su proyecto reformista y nacionalista a } \\
\text { través de los deportes, así como también los principales debates entre columnistas deportivos, periodistas, } \\
\text { médicos, profesores de educación física y dirigentes políticos en las páginas de la revista Los Sports sobre el rol del } \\
\text { Estado como garante de la actividad física en Chile. }\end{array}$ & Políticas públicas \\
\hline $\begin{array}{l}\text { Castillo-Retamal et al., } \\
\qquad(2020)\end{array}$ & $\begin{array}{c}\text { Revisión de documentos } \\
\text { ministeriales, leyes e } \\
\text { investigaciones de bases de datos. }\end{array}$ & Manuscrito & $\begin{array}{c}\text { Analizar el proceso de formación de profesores en Chile y particularmente, el desarrollo histórico de la disciplina } \\
\text { Educación Física (EF). }\end{array}$ & Políticas públicas \\
\hline Curilem (2017) & 17 profesores PEF y 174 escolares. & Tesis doctoral & $\begin{array}{c}\text { Diseñar y validar una nueva prueba del Sistema de calidad de la Educación (SIMCE) que pueda satisfacer la } \\
\text { necesidad de integrar todas las dimensiones y variables que le pertenecen a la disciplina. }\end{array}$ & Políticas públicas \\
\hline Flores et al., (2019) & 141 estudiantes de PEF. & Manuscrito & $\begin{array}{l}\text { Conocer las propiedades psicométricas del cuestionario de Motivos de Deserción universitaria de los estudiantes } \\
\text { de Educación Física y determinar los principales motivos de deserción de los estudiantes de la carrera de } \\
\text { Pedagogía en Educación Física de la Universidad Católica Silva Henríquez de Santiago de Chile. }\end{array}$ & Educación superior \\
\hline Flores et al., (2020a) & 23 universidades. & Manuscrito & $\begin{array}{l}\text { Los perfiles de egreso de la carrera de Pedagogía en Educación Física de Chile, con el fin de identificar las } \\
\text { competencias que aparecen con mayor frecuencia y cuáles son las más reconocidas tanto en las instituciones } \\
\text { públicas como privadas a nivel nacional. }\end{array}$ & Educación superior \\
\hline Flores et al., (2020b), & 542 estudiantes de PEF. & Manuscrito & Conocer el nivel de satisfacción de las clases virtuales de los estudiantes de Educación Física. & Educación superior \\
\hline Flores et al., (2020c) & 908 estudiantes de PEF. & Tanuscrito & Determinar la percepción institucional de los estudiantes de Pedagogía en Educaci & Educación superior \\
\hline Gálvez et al., (2016) & 212 estudiantes de PEF. & Manuscrito & $\begin{array}{c}\text { Conocer las propiedades psicométricas de La Escala de Mediadores Motivacionales en el Deporte (EMMD) en } \\
\text { estudiantes de educación física de Santiago de Chile y determinar los niveles de satisfacción de las necesidades } \\
\text { motivacionales }\end{array}$ & Educación superior \\
\hline Giakoni et al., (2021) & 224 escolares. & Manuscrito & $\begin{array}{l}\text { Determinar la influencia que tiene el volumen de clases: dos o cuatro horas de Educación Física semanal, sobre la } \\
\text { composición corporal, la condición física y nivel de actividad física en escolares chilenos }\end{array}$ & Escolar \\
\hline González et al., (2020) & 214 estudiantes de PEF. & Manuscrito & $\begin{array}{r}\text { Realizar la construcción y validación de un instrumento } \\
\text { Educación fí. }\end{array}$ & Educación superio \\
\hline Jiménez et al., (2013) & 12 universidades. & Manuscrito & $\begin{array}{l}\text { Realizar una revisión crítica de los diseños curriculares, materias, contenidos y descriptores de los Centros de } \\
\text { Formación Docente de Educación Física en Chile utilizando los parámetros principios y fundamentos de la } \\
\text { Praxiología Motriz o ciencia de la acción motriz; y de los fundamentos de la sociología de la Educación y del } \\
\text { Currículo. }\end{array}$ & Educación superio \\
\hline López (2016) & $\begin{array}{l}\text { Revisión de documentos } \\
\text { ministeriales, leyes e } \\
\text { investigaciones de bases de datos. }\end{array}$ & anuscrito & Realizar una reflexión sobre buen vivir, sistema escolar y educación física en Chile una realidad por construir. & Políticas públicas \\
\hline Luarte et al., (2014) & 174 escolares. & Manuscrito & $\begin{array}{l}\text { Conocer si los pre-escolares de la comuna de Chiguayante que no han recibido intervención } \\
\text { educación física presentan un nivel de desarrollo motor grueso de acuerdo a su }\end{array}$ & Escolar \\
\hline Maureira (2013) & 44 estudiantes de PEF. & Manuscrito & $\begin{array}{r}\text { Determinar los niveles de validez de constructo y de confiabilidad de cada sub-escala de los es } \\
\text { del CHAEA en una muestra de estudiantes de educación física de Chile. }\end{array}$ & Educación superior \\
\hline Maureira et al., (2018) & 141 estudiantes de PEF. & Manuscrito & $\begin{array}{r}\text { Conocer las propiedades psicométricas del Inventario } \\
\text { Felder-Silverman en una muestra de }\end{array}$ & Educación superior \\
\hline $\begin{array}{l}\text { Maureira-Cid et al., } \\
\text { (2021a) }\end{array}$ & 116 estudiantes de PEF. & Manuscrito & $\begin{array}{r}\text { Relacionar los estilos de aprendizaje, la habilidad emocional, las inteligencias m } \\
\text { través de la mirada en una selección de estudiantes de educaci }\end{array}$ & Educación superior \\
\hline $\begin{array}{l}\text { Maureira-Cid et al., } \\
\text { (2021b) }\end{array}$ & 440 estudiantes de PEF. & Manuscrito & $\begin{array}{r}\text { Describir la prevalencia de neuromitos el conocimiento general de neuroc } \\
\text { Educación Física de diversas universidades d }\end{array}$ & Educación superior \\
\hline Maureira $(2)$ & 5 estudiantes de PEF. & anuscrito & Describir los conocimientos biológicos de la homosexu & E \\
\hline Martínez (2011) & $\begin{array}{l}\text { Revisión de documentos } \\
\text { ministeriales, leyes e } \\
\text { investigaciones de bases de datos. }\end{array}$ & Manuscrito & $\begin{array}{c}\text { Historiar la implementación y el desarrollo de la educación física (EF) como una disciplina y práctica pedagógica } \\
\text { en Chile entre los años } 1889 \text { y 1920, la cual tuvo en consideración la obligatoriedad de esta disciplina en contextos } \\
\text { escolares para el fortalecimiento de sus cuerpos infantiles como una salud y una herramienta para el desarrollo de } \\
\text { la modernización en el país }\end{array}$ & Políticas públicas \\
\hline Martínez (2012) & $\begin{array}{l}\text { Revisión de documentos } \\
\text { ministeriales, leyes e } \\
\text { investigaciones de bases de datos. }\end{array}$ & Manuscrito & $\begin{array}{c}\text { Historiar la implementación y el desarrollo de la educación física (EF) como una disciplina y práctica pedagógica } \\
\text { en Chile entre los años } 1889 \text { y 1920, la cual tuvo en consideración la obligatoriedad de esta disciplina en contextos } \\
\text { escolares para el fortalecimiento de sus cuerpos infantiles como una salud y una herramienta para el desarrollo de } \\
\text { la modernización en el país }\end{array}$ & Políticas públicas \\
\hline Moreno et al., (2014) & $\begin{array}{l}25 \text { documentos que abarcan tanto } \\
\text { la educación básica como la } \\
\text { educación media. }\end{array}$ & Manuscrito & $\begin{array}{c}\text { Análisis de los programas existentes desde } 1^{\circ} \text { básico a } 4^{\circ} \text { medio de enseñanza científico humanista, jornada escolar } \\
\text { completa, horas de libre disposición y técnico profesional, donde encontraron que los programas estaban basados } \\
\text { en ideas centrales para moverse mejor, salud con enfoque biomédico, deportes y dimensiones afectivas y sociales } \\
\text { del ser humano referidas al desarrollo personal y a la formación ética intelectual }\end{array}$ & Políticas públicas \\
\hline Navarro et al., (2021) & $\begin{array}{l}10 \text { estudiantes y } 10 \text { profesores de } \\
\text { PEF. }\end{array}$ & Manuscrito & $\begin{array}{c}\text { Identificar los obstáculos y desafíos que profesores y estudiantes de la carrera de pedagogía en educación física de } \\
\text { una universidad en Chile, manifiestan de las Estrategias Pedagógicas Integrada (EPI). }\end{array}$ & Educación superio \\
\hline Rannau (2020a) & $\begin{array}{l}\text { Documentos ministeriales, leyes y } \\
\text { SIMCE. }\end{array}$ & Manuscrito & $\begin{array}{c}\text { Reflexionar sobre la Educación Física en Chile: hacia una mirada transdisciplinariedad desde el currículum y la } \\
\text { colaboración pedagógica }\end{array}$ & Políticas públicas \\
\hline Reyno (2010b) & 46 programas. & Manuscrito & $\begin{array}{c}\text { Extraer información acerca de los contenidos declarados en los programas relacionados con el Área de Expresión } \\
\text { Motriz (AEM) de las Carreras de Pedagogía en Educación Física de las Universidades, cuyos proyectos de } \\
\text { Formación Inicial Docente fueron aprobados por el Ministerio de Educación. }\end{array}$ & Educación superior \\
\hline Reyno (2010) & 249 profesores de PEF. & Manuscrito & $\begin{array}{l}\text { Analizar la opinión acerca de los objetivos y contenidos de la Unidad «Deportes y actividades de autosuperación y } \\
\text { de expresión motriz»y de las Actividades Expresivo Motrices (AEM), emitida por profesores de Educación Física } \\
\text { (EF) en ejercicio, en el año 2004 en Chile. }\end{array}$ & Escolar \\
\hline Reyno (2011) & 248 profesores de $\mathrm{EF}$ & Manuscrito & $\begin{array}{l}\text { Analizar el nivel de preparación de los profesores de Educación Física (EF) en los contenidos relacionados con las } \\
\text { Actividades Expresivo Motrices, la Gimnasia Rítmica y su aplicación en el medio escolar }\end{array}$ & Escolar \\
\hline $\begin{array}{l}\text { Riobó y Villarroel } \\
\text { (2019) }\end{array}$ & $\begin{array}{l}\text { Revisión de documentos } \\
\text { ministeriales, leyes e } \\
\text { investigaciones de bases de datos. }\end{array}$ & Manuscrito & $\begin{array}{c}\text { Buscó presentar y contextualizar la fuente "Aspectos de la educación física", de Luis Bisquertt, poniendo énfasis en } \\
\text { la búsqueda del mejoramiento racial en Chile de 1930, a partir de la educación física eugénica, cuyo paradigma } \\
\text { corporal serían las estatuas clásicas helénicas. }\end{array}$ & Políticas públicas \\
\hline $\begin{array}{l}\text { Rivera y Henríquez } \\
\text { (2018) }\end{array}$ & $\begin{array}{l}\text { Revisión de documentos } \\
\text { ministeriales, leyes e } \\
\text { investigaciones de bases de datos. }\end{array}$ & Manuscrito & $\begin{array}{l}\text { Estudiar la construcción disciplinar de la asignatura de Educación Física en Chile desde la región de La Araucanía } \\
\text { considerando su relación y efectos sobre la población indígena de la región antes señalada. }\end{array}$ & Políticas públicas \\
\hline $\begin{array}{l}\text { Rivera y Henríquez- } \\
\text { Alvear (2019) }\end{array}$ & Documentos de Murillo y Aguirre. & Manuscrito & $\begin{array}{l}\text { Analizar los documentos de Murillo (1872) y Aguirre (1886) con el fin de evidenciar la influencia programática de } \\
\text { los escritos del siglo XIX sobre los actuales lineamientos de la asignatura de Educación Física en Chile. }\end{array}$ & Políticas públicas \\
\hline $\begin{array}{l}\text { Rodríguez-Rodríguez } \\
\text { (2016) }\end{array}$ & $\begin{array}{l}\text { Revisión de documentos } \\
\text { ministeriales, leyes e } \\
\text { investigaciones de bases de datos. }\end{array}$ & Manuscrito & $\begin{array}{l}\text { Acentuar la problemática y plantear ideas que permitan una coherencia entre los elementos en juego para una } \\
\text { Educación Física de calidad, integrando propuestas sobre las variables que influyen en la mejora de la disciplina a } \\
\text { nivel escolar. }\end{array}$ & Políticas públicas \\
\hline Veliz et al., (2015) & $\begin{array}{l}\text { Revisión de documentos } \\
\text { ministeriales, leyes e } \\
\text { investigaciones de bases de datos. }\end{array}$ & Manuscrito & Recopilación de antecedentes importantes de la historia de la Educación Física en Chile. & Políticas públicas \\
\hline
\end{tabular}
PEF: Pedagogía en Educación Física.

\section{Contextos escolares}

Giakoni et al., (2021), realizaron una investigación la cual tuvo por objetivo determinar la influencia que tiene el volumen de clases: dos o cuatro horas de Educación Física semanal, sobre la composición corporal, la condición física y nivel de actividad física en escolares chilenos. En esta intervención participaron un total de 
224 escolares de $7^{\circ}$ y $8^{\circ}$ básico $(2$ horas $\mathrm{n}=81$ y 4 horas $\mathrm{n}=163$ ). Los instrumentos utilizados para la medición de las variables fueron una balanza, para el peso; un tallímetro. para la estatura y obtención del IMC; una cinta para la medición del perímetro de cintura; prueba de 20 metros (protocolo EUROFIT) para evaluar la potencia aeróbica máxima; salto de longitud para medir la fuerza del tren inferior; dinamómetro manual para medir la fuerza del tren superior; y se utilizó un acelerómetro triaxial Actigraph modelo GT3X para medir el nivel de actividad física. Los investigadores concluyeron que ambos grupos de estudiantes (2 horas y 4 horas) cumplieron con las recomendaciones de AF de la OMS, sin embargo, señalaron que realizar al menos cuatro horas semanales de Educación Física, ejerce efectos positivos sobre la condición física y en factores como la composición corporal.

Por otra parte, la investigación que desarrollaron Luarte et al., (2014), tuvo por objetivo conocer si los pre-escolares de la comuna de Chiguayante que no han recibido intervención de profesores de educación física presentan un nivel de desarrollo motor grueso de acuerdo a su edad. La muestra estuvo compuesta por 173 escolares, los cuales fueron sometidos al test de desarrollo motor grueso de ULRICH (2000). Los principales resultados obtenidos fueron que un $11,7 \%$ de la muestra estuvo categorizada en muy pobre y un 32,9\% en la categoría pobre. Los investigadores concluyeron que el nivel de desarrollo motor grueso esperado para la edad de la muestra se encontró en las categorías Bajo la edad, Pobre y Muy pobre.

Reyno (2010), realizó una investigación que tuvo por objetivo analizar la opinión acerca de los objetivos y contenidos de la Unidad «Deportes y actividades de autosuperación y de expresión motriz» y de las Actividades Expresivo Motrices (AEM), emitida por profesores de Educación Física (EF) en ejercicio, en el año 2004 en Chile. La muestra estuvo compuesta por 249 profesores que impartían EF en los cursos de secundaria, en establecimientos educativos del país. La autora concluyó que los profesores de EF de secundaria se centran de manera principal en la aptitud física, salud y calidad de vida.

Retomando nuevamente a Reyno (2011), en su investigación la cual tuvo por objetivo general analizar el nivel de preparación de los profesores de Educación Física (EF) en los contenidos relacionados con las Actividades Expresivo Motrices, la Gimnasia Rítmica y su aplicación en el medio escolar. La muestra estuvo compuesta por 248 profesor de EF de secundaria de estable- cimientos municipales, particular subvencionado, particular pagado y/o corporación. La investigadora concluyó que los profesores recibieron contenidos de AEM en su proceso de formación, en asignaturas de carácter obligatorio y mixtos (cursos con participación de hombres y mujeres), desarrollándose contenidos prioritarios de folclore y gimnasia artística.

\section{Educación superior}

Maureira-Cid et al., (2021), realizaron una investigación la cual tuvo por objetivo relacionar los estilos de aprendizaje, la habilidad emocional, las inteligencias múltiples y la detección emocional a través de la mirada en una selección de estudiantes de educación física de Chile. En este trabajo de investigación participaron un total de 116 estudiantes de Educación Física de una universidad privada de Santiago de Chile. El instrumento utilizado fue el cuestionario, en su versión reducida del Honey-Alonso de Estilos de Aprendizaje (CHAEA-36) de 36 preguntas con respuesta dicotómica (sí - no), el cual entrega puntuaciones en cada uno de los estilos de aprendizaje (activo, reflexivo, teórico y pragmático); para la habilidad emocional se utilizó una versión adaptada para educación física del inventario de Rego \& Fernandes (2005), dicha versión cuenta con 32 ítems escala tipo Likert con 5 opciones (desde 1 totalmente en desacuerdo hasta 5 totalmente de acuerdo); y Para las habilidades múltiples se utilizó el Multiple Intelligences Developmental Assessment Scales (MIDAS) que es un instrumento para medir el desarrollo de las inteligencias múltiples en adultos, mediante preguntas con 6 alternativas de respuesta; y para la detección emocional a través de la mirada se utilizó la prueba de la mirada de Baron et al. (2001) que consta de 36 fotografías en blanco y negro, de ojos y cejas de personas de ambos sexos, donde el evaluado debe escoger , entre cuatro opciones, la que mejor represente el estado mental de la imagen. Los investigadores concluyeron que los cuatros estilos de aprendizaje se relacionan positivamente o negativamente con alguna habilidad emocional de relación positiva o negativa con alguna inteligencia múltiples y que el reconocimiento emocional a través de la mirada se relaciona negativamente con las habilidades lógicas.

Por otra parte, la investigación realizada por Maureira-Cid et al., (2021), tuvo por objetivo describir la prevalencia de neuromitos el conocimiento general de neurociencia de estudiantes de Pedagogía en Educación Física de diversas universidades de Chile. La mues- 
tra estuvo confirmada por 440 estudiantes universitarios. El cuestionario utilizado fue la versión en español del Cuestionario de prevalencia de neuromitos desarrollado por Dekker et al. (2012). Los investigadores concluyeron que los estudiantes universitarios de Educación Física en Chile, poseen neuromitos similares a los reportados en estudiantes y profesores de otras carreras de pedagogía en Chile.

A su vez, el trabajo realizado por Maureira (2021), que tuvo por objetivo describir los conocimientos biológicos de la homosexualidad y transexualidad de estudiantes de Educación Física. La muestra estuvo compuesta por 305 estudiantes de Pedagogía en Educación Física de tres universidades chilenas. Para la investigación se construyó y validó un cuestionario diseñado ad hoc a 305 estudiantes de Pedagogía en Educación Física de tres universidades de Chile. El autor concluyó que los estudiantes de Pedagogía en Educación Física, en 14 de los 18 ítems desarrollados, respondieron «No saber» sobre temas relacionados con Conocimiento Biológicos de la Homosexualidad y Transexualidad (CBHT).

En el trabajo realizado por Navarro et al., (2021), tuvo por objetivo identificar los obstáculos y desafíos que profesores y estudiantes de la carrera de pedagogía en educación física de una universidad en Chile, manifiestan de las Estrategias Pedagógicas Integrada (EPI). La muestra estuvo compuesta por 10 estudiantes y 10 profesores de la carrera de pedagogía en Educación Física de una universidad en Chile. La recolección de los datos se llevó a cabo a través de una entrevista semiestructurada a cada uno de los participantes. Los investigadores concluyeron que según lo planteado por los sujetos que participaron del estudio, señalaron que las estrategias pedagógicas integradas son una necesidad a nivel del contexto universitario chileno, más allá de las miradas nomotéticas de productividad que se visualizan.

Flores et al. (2020), desarrollaron una investigación, la cual tuvo por objetivo determinar los perfiles de egreso de la carrera de Pedagogía en Educación Física de Chile, con el fin de identificar las competencias que aparecen con mayor frecuencia y cuáles son las más reconocidas tanto en las instituciones públicas como privadas a nivel nacional. La muestra estuvo constituida por 23 universidades que dictan la carrera de Pedagogía en Educación Física. Los resultados mostraron que las principales competencias fueron por parte de las universidades: La interpretación crítica del conocimiento y/o reflexión sobre la práctica pedagógica y profesional, el diseño y aplicación actividades de aprendizaje y evaluación; y el trabajo colaborativo y liderazgo. Los investigadores concluyeron que se requieren futuras investigaciones que permitan la identificación de los estándares pedagógicos y disciplinares actuales de las carreras de Pedagogía en Educación Física en Chile, a través de documentos o perfiles de egreso de manera íntegra y completa.

Por otra parte, la investigación de Flores et al., (2020), desarrollaron una investigación que tuvo por objetivo conocer el nivel de satisfacción de las clases virtuales de los estudiantes de Educación Física. La muestra estuvo compuesta por 542 estudiantes universitarios de Educación Física de diferentes universidades en Chile. Se adaptó y validó la escala de Satisfacción clases online. Los investigadores concluyeron que existe una resistencia para el desarrollo de las clases virtuales por parte de los estudiantes, debido a que se encontraron diferencias significativas entre las asignaturas teóricas y prácticas.

A su vez, Flores et al., (2020), realizaron una investigación, cuyo objetivo fue determinar la percepción institucional de los estudiantes de Pedagogía en Educación Física de Chile. La muestra estuvo compuesta por 908 estudiantes universitarios de pedagogía en Educación Física. Se aplicó una escala de percepción de la carrera de pedagogía en Educación Física. Entre los resultados, se encontraron diferencias significativas entre universidades públicas y privadas en la gestión universitaria y protestas estudiantiles. Los investigadores concluyeron que, por parte de los estudiantes universitarios, señalaron que la percepción institucional tiene mayor relevancia es la gestión académica.

González et al., (2020), realizaron la construcción y validación de un instrumento para valorar la calidad de la formación de profesores de Educación Física en Chile. La investigación tuvo por objetivo la determinación de protocolos de elaboración (consulta de expertos nacionales $n=8$ e internacionales $n=6$, respecto a aspectos formales, metodológicos y selección de ítems por dimensión (Expresión, Didáctica, Gestión, Entrenamiento, Deportes, Recreación, Salud y Primeros Auxilios). la muestra estuvo compuesta por 214 estudiantes de 6 universidades chilenas. Hechas las correspondientes correcciones a partir de la evidencia, se configuró una versión final del instrumento con 70 ítems, cuya caracterización psicométrica presenta una consistencia interna de .778, un índice promedio de dificultad adecuada de 0,59, un índice promedio de discriminación alta de 0,39 y un coeficiente de discriminación bueno de 0,25 , lo que permite señalar que la prueba es ade- 
cuada para medir adquisición de saberes en el proceso de formación. en relación con los ítems de expresión, didáctica, gestión, entrenamiento, deportes, recreación, salud y primeros auxilios. Los autores concluyeron que el instrumento permitió establecer de manera objetiva el grado de dominio del conocimiento de los estudiantes universitarios de Pedagogía en Educación Física en relación a las bases curriculares, planes y programas del Ministerio de Educación, sin embargo, este instrumento debe ser actualizado de manera constante para dar respuesta de manera integral al futuro profesor.

A su vez, una investigación realizada por Flores et al. (2019), la cual tuvo los siguientes objetivos conocer las propiedades psicométricas del cuestionario de Motivos de Deserción universitaria de los estudiantes de Educación Física y determinar los principales motivos de deserción de los estudiantes de la carrera de Pedagogía en Educación Física de la Universidad Católica Silva Henríquez de Santiago de Chile. La muestra estuvo compuesta por 141 estudiantes de pedagogía en Educación Física. Se creo un cuestionario de motivos de deserción universitaria. Los investigadores concluyeron que los estudiantes de Educación Física podrían abandonar la carrera debido a posibles factores como el descontento a la carrera y los compromisos adquiridos por la universidad.

Maureira et al., (2018), realizaron una investigación que tuvo por objetivo conocer las propiedades psicométricas del Inventario de Estilos de Aprendizaje de Kolb y del Cuestionario de Felder-Silverman en una muestra de estudiantes de Educación Física de Chile. La muestra estuvo compuesta por 141 estudiantes de Educación física. Se utilizaron dos instrumentos, uno de ellos fue el Inventario de Estilos de Aprendizaje de Kolb y del Cuestionario de Felder-Silverman. En sus resultados, los investigadores señalaron que ambos cuestionarios poseen índices adecuados de validez, pero los índices de confiabilidad son bajos en los niveles aceptados en todas las sub-escalas. Los autores concluyeron que es recomendable la realización de más estudios con muestras de otras universidades para la confirmación de las propiedades psicométricas del instrumento aplicado en estudiantes universitarios.

Gálvez et al., (2016) realizaron una investigación que tuvo los siguientes objetivos, conocer las propiedades psicométricas de La Escala de Mediadores Motivacionales en el Deporte (EMMD) en estudiantes de educación física de Santiago de Chile y determinar los niveles de satisfacción de las necesidades motivacionales. La muestra estuvo compuesta por 212 estudiantes de Educación física de tres universidades, donde se les aplicó la el EMMD. Los estudiantes señalaron que sienten confianza en sus compañeros de entrenamiento, ya que son amables, confían en su fuerza y se sienten satisfechos al momento de seleccionar alguna actividad para su entrenamiento. Los investigadores concluyeron que el instrumento EMMD es válido y confiable para estudiantes de Educación Física en Chile.

A su vez, Maureira (2013) realizó una investigación que tuvo por objetivo determinar los niveles de validez de constructo y de confiabilidad de cada sub-escala de los estilos de aprendizaje del CHAEA en una muestra de estudiantes de Educación Física de Chile. La muestra estuvo compuesta por 44 estudiantes universitarios de pedagogía en Educación Física. El autor concluyó que los valores obtenidos KMO fueron aceptables en todos los estilos e ítems de cada sub-escala, lo cual presentó validez al ser aplicado en los estudiantes de pedagogía en Educación Física.

En el trabajo realizado Reyno (2010), el cual tuvo por objetivo extraer información acerca de los contenidos declarados en los programas relacionados con el Área de Expresión Motriz (AEM) de las Carreras de Pedagogía en Educación Física de las Universidades, cuyos proyectos de Formación Inicial Docente fueron aprobados por el Ministerio de Educación. Se analizaron 46 programas de estudio, en la cual se aplicó una técnica de análisis semántico, considerando dimensiones tales como «identificación», «unidades»y «contenido». La autora concluyó que en la Formación Inicial de las carreras de Educación Física en Chile las asignaturas relacionadas con las AEM se agruparon bajo las denominaciones de danza, expresión y creatividad, gimnasia, folclore y ritmo.

Finalmente, Jiménez et al., (2013) realizaron una investigación cuyo objetivo fue realizar una revisión crítica de los diseños curriculares, materias, contenidos y descriptores de los Centros de Formación Docente de Educación Física en Chile utilizando los parámetros principios y fundamentos de la Praxiología Motriz o ciencia de la acción motriz; y de los fundamentos de la sociología de la Educación y del Currículo. Los autores utilizaron como referencia el Plan Bolonia (1999) para el Espacio Europeo de Educación Superior, el Espacio Iberoamericano del Conocimiento (EIC, 2005) y el Proyecto Tuning en Latino América (2004-2007). Los autores concluyeron que la formación de profesores en Chile depende de cada institución conforme a la misión y visión de cada una, además la Praxiología Motriz no es referente en los programas de formación docente en 
las carreras de Pedagogía en Educación Física en Chile.

\section{Políticas públicas}

Rannau (2020), realizó una investigación reflexiva sobre la Educación Física en Chile: hacia una mirada transdiciplinaria desde el currículum y la colaboración pedagógica. En la investigación se realizó la reflexión desde cinco temáticas, estas son: «La Educación Física como práctica de intervención en el currículum nacional», donde aborda desde la reflexión la Ley 20.370, y la manera que la Educación Física y Salud es una asignatura central de la educación escolar desde la propuesta del Ministerio de Educación y la evaluación SIMCE; «Desde un currículum fragmentado hacia una mirada globalizadora», estableciendo una escuela parcelada y sistematizada, dado que una educación debe tener por función estimular la cognición, personalidad y aspectos conductuales, además de considerar la adaptación en el medio físico y social del alumnado; «La necesaria permeabilidad de los saberes disciplinares en la experiencia motriz», desde una mirada filosófica, desde el ser y metódica, las áreas disciplinares, deben permitir la transferencia e integración de saberes, considerando actitudes, emociones y valores en la formación y no solo certificar una competencia motriz; Inter o transdisciplinariedad en la Educación Física, ¿qué posibilidades nos ofrece nuestra realidad?, desde la Educación Física, se hace necesario replantear una pedagogía de la motricidad y potenciar todas las potencialidades del ser humano desde la antropología-cultural, permitiendo la globalización del abordaje de competencias sociales y ciudadanas, vinculando el currículo y el trabajo comunitario; «La oportunidad en la escuela: sobre la docencia, lo motriz y la colaboración», el rol docente es fundamental, dado que permite la interpretación de la didáctica del currículo y de esta manera no se transforma es un adiestramiento técnico. El autor concluyó que el sistema educativo en Chile, dado a sus disciplinas curriculares, se encuentra fragmentado, ya que no permite la formación de un ser integral, llevando a la Educación Física al cumplimiento de una competencia motriz.

Castillo-Retamal et al., (2020), realizaron una revisión histórica de la formación de profesores de Educación Física en Chile, esta mirada histórica tuvo por objetivo analizar el proceso de formación de profesores en Chile y particularmente, el desarrollo histórico de la disciplina Educación Física (EF). Los resultados indicaron que la EF transitan desde una perspectiva técnica hacia modelos pedagógicos. Los investigadores concluyeron que la EF en Chile debe permitir el desarrollo humano, desde la integración de manera adecuada de la actividad física, deporte y la salud, siempre dando respuesta a los requerimientos ministeriales desde los estándares pedagógicos y disciplinares.

La investigación desarrollada por Rivera y Henríquez-Alvear (2019), tuvo por objetivo analizar los documentos de Murillo (1872) y Aguirre (1886) con el fin de evidenciar la influencia programática de los escritos del siglo XIX sobre los actuales lineamientos de la asignatura de Educación Física en Chile. Los investigadores concluyeron que los propósitos de la Educación Física actual mantienen una orientación hacia lo biomédico, higiénico y moral, buscando el bienestar de los sujetos y desarrollo integral de los mismos.

Por otra parte, Rivera y Henríquez (2018), tuvo por objetivo estudiar la construcción disciplinar de la asignatura de Educación Física en Chile desde la Región de La Araucanía considerando su relación y efectos sobre la población indígena de la región antes señalada. Los autores concluyeron que fue posible observar las influencias extranjeras médico-higiénicas, gimnastas y deportivas, considerando de manera histórica el rol civilizador, moralizador y normalizador de la población en general, las poblaciones indígenas no fueron consideradas históricamente desde la Educación Física y Salud.

En su tesis doctoral, Curilem (2017), tuvo por objetivo general diseñar y validar una nueva prueba del Sistema de calidad de la Educación (SIMCE) que pueda satisfacer la necesidad de integrar todas las dimensiones y variables que le pertenecen a la disciplina. Su propuesta consistió en tres ámbitos, el primero relacionado con evaluaciones motrices, el segundo relacionado con autoevaluación de la gestión docente y el tercero relacionado con una encuesta de opinión de los escolares. El autor concluyó los siguientes puntos relevantes, la propuesta desarrollada, válida y fiable, relacionada con la prueba escrita para los escolares se encentraron alineados con los planes y programas, permitiendo dar cumplimiento a lo solicitado por el Ministerio de Educación, la evaluación escrita para los docentes permitió la obtención de información de calidad de la educación física desde una mirada integral y general, dado que una evaluación del contexto escolar permite añadir información relevante de los múltiples factores involucrados en la propuesta.

Veliz et al., (2015), desde una breve historia de la Educación Física en Chile, se realizó una recopilación de antecedentes importantes de la historia de la Educa- 
ción Física en Chile, desde la época de la colonia, reformas educativas, aplicación de la evaluación SIMCE, hasta la actualidad, además de interiorizar la fundación del primer Instituto Superior de Educación Física. Los investigadores concluyeron que la Educación Física ha transitado por diversas transformaciones, desde la preparación para conflictos bélicos, a la actividad física para la salud y el bienestar en contextos escolares.

La propuesta desarrollada por López (2016), tuvo por objetivo realizar una reflexión sobre buen vivir, sistema escolar y Educación Física en Chile una realidad por construir. El cual evidenció las transformaciones de la Educación Física en Chile, llevando la palabra «salud» a contextos escolares para dar respuesta a los lineamientos de la Organización Mundial de la Salud (OMS) para la mantención de la salud y a las prioridades de los gobiernos de turno. El investigador señaló que, para la mantención de la salud, existen variables o factores relacionados con la armonía y relación con su entorno, variables objetivas y subjetivas de la persona, demandas sociales en torno a la cultura y educación del alumnado, y procesos para la adquisición de hábitos desde el núcleo familiar y la escuela.

Además, Martínez (2011), desarrolló una investigación que tuvo por objetivo historiar la implementación y el desarrollo de la Educación Física (EF) como una disciplina y práctica pedagógica en Chile entre los años 1889 y 1920, la cual tuvo en consideración la obligatoriedad de esta disciplina en contextos escolares para el fortalecimiento de sus cuerpos infantiles como una salud y una herramienta para el desarrollo de la modernización en el país. De esta manera, Martínez (2012), nos indica que la Pedagogía en Educación Física entre los años 1890 y 1920 tuvo un fuerte vínculo entre la medicina y la pedagogía, esto permitió que las políticas públicas en nuestro país tuvieran un componente significativo, debido que los médicos y los profesores promovían el concepto de «actividad física - salud» con el fin de desarrollar hábitos preventivos y de higiene para la prevención de enfermedades durante ese periodo. El autor concluyó que la actividad física pasó a ser considerada políticamente como un servicio social, donde el Estado debía proporcionar a los ciudadanos para su beneficio y bienestar personal.

Moreno et al., (2014), realizaron un análisis de los programas existentes desde $1^{\circ}$ básico a $4^{\circ}$ medio de enseñanza científico humanista, jornada escolar completa, horas de libre disposición y técnico profesional, donde encontraron que los programas estaban basados en ideas centrales para moverse mejor, salud con enfo- que biomédico, deportes y dimensiones afectivas y sociales del ser humano referidas al desarrollo personal y a la formación ética intelectual. Los autores concluyeron que la Educación Física en Chile tuvo un tránsito por diversos propósitos para el desarrollo de la población, desde la promoción de la salud, productividad, militarización, higiene, seguridad y deportivización, además de ser considerada un derecho social, sin embargo, se encuentra sustentada en fundamentos científicos fisiológicos, biomédicos o biomecánicos, dejando de lado las actualices inquietudes sociales.

Por otra parte, Riobó y Villarroel (2019) buscaron contextualizar la Educación Física desde la «belleza plástica, eugenesia y educación física en Chile: presentación de la fuente» desde la mirada de Luis Bisquertt (médico y profesor de Educación Física) en el año1930, esta investigación nos señala en un viaje por Europa para el análisis de la Educación Física, menciona que los «Aspectos de la educación física» deben ser encarnados y prefiguradas varias de las posiciones que se ven vinculadas a una concepción eugénica de la Educación Física se pone contraste con la comprensión guerrera de la Educación Física, donde llegará a su cúspide años más tarde en la Alemania Nazi ya que se poseía el canon clásico como modelo de cuerpo ideal. El Dr. Bisquertt a su vez, declaró que la Educación Física debiera ser nacional socialista siendo fundamental en la formación escolar, así como por su carácter social y obligatorio, ésta debía ser emulada por todas las naciones civilizadas, aunque el objetivo belicoso y racista que persigue sea condenado, considerando el ámbito gimnástico y el modelo espartano.

Acuña (2020), realizó un trabajo de investigación que buscó explorar los esfuerzos institucionales del Gobierno ibañista por legitimar su proyecto reformista y nacionalista a través de los deportes, así como también los principales debates entre columnistas deportivos, periodistas, médicos, profesores de Educación Física y dirigentes políticos en las páginas de la revista Los Sports sobre el rol del Estado como garante de la actividad física en Chile. El autor señaló que en el primer mandato del presidente Carlos Ibáñez impulso políticas deportivas nacionales, promulgando una Ley obligatoria para la Educación Física en Chile en el año 1929. El autor concluyó que el presidente Ibáñez, no logró materializar su programa deportivo, a pesar de su intenso reformismo entre los años 1927 y 1931, pero tuvo un fuerte impacto cultural para la inclusión de los deportes femeninos.

Finalmente, en la investigación desarrollada por 
Rodríguez-Rodríguez (2016), la cual intentó acentuar la problemática y plantear ideas que permitan una coherencia entre los elementos en juego para una Educación Física de calidad, integrando propuestas sobre las variables que influyen en la mejora de la disciplina a nivel escolar. El autor profundizo temáticas relacionadas con sistemas de evaluación de la calidad de la educación, SIMCE de Educación Física desde el origen de la Ley del Deporte (2001), Sistema de evaluación de la calidad de la condición física del Centro de Control y Prevención de los Estados Unidos (CDC) y la creación del PECAT por parte del CDC en el 2006. El autor concluyó que desde la evaluación SIMCE de Educación Física, su principal dificultad ha sido articular las tres dimensiones del ser humano integral, desde lo corporal, racional y emocional, debido a que esta evaluación solo evalúa una de estas dimensiones, no considerando factores socioemocionales y socioeducativos.

\section{Discusión}

De las 31 investigaciones, que cumplieron con nuestros criterios de selección, fueron clasificadas en tres grupos, investigaciones relacionadas con «Contexto escolar» (4 artículos), investigaciones en «Educación Superior» (14 artículos) e investigaciones relacionadas con «Políticas Públicas» (13 artículos).

Los artículos en «Contextos escolares», desarrollaron propuestas de intervención en escolares del sistema educativo, donde se trató de determinar la influencia de la clase de Educación Física (Giakoni, Paredes y Duclos-Bastías, 2021), conocer el nivel de desarrollo motor grueso en pre escolares (Luarte, Poblete y Flores, 2014), analizar la opinión acerca de los objetivos y contenidos de la Unidad «Deportes y actividades de autosuperación y de expresión motriz» y de las Actividades Expresivo Motrices (AEM), emitida por profesores de EF (Reyno, 2010) y analizar la preparación de los profesores de EF en contenidos relacionados con AEM (Reyno, 2011). De las 4 investigaciones, se evidenció que en dos de ellas la muestra estuvo compuesta por escolares, a los que se evaluó desde la perspectiva físico-motriz y las otras dos investigaciones recoge información desde el profesorado de la asignatura, evidenciando sus competencias sobre las AEM. Sin lugar a duda, en la «Educación superior» es donde descansa la formación inicial docente del profesorado de Educación Física. La muestra de estos artículos estuvo compuesta por estudiantes universitarios de las carreras de Pedagogía en Educación Física (Flores et al. 2019; Flores et al.,
2020; Flores et al., 2020; Galvez et al., 2016; Maureira, 2013; Maureira et al., 2018; Maureira et al., 2021; Maureira, 2021; Maureira et al., 2021), estas investigaciones tuvieron objetivos relacionados con la validación de instrumentos de medición de la percepción de satisfacción relacionados con el desarrollo de las clases de Pedagogía de manera online, relacionar estilos de aprendizaje, prevalencia de neuromitos, conocimientos biológicos de la homosexualidad y transexualidad, propiedades psicométricas de un cuestionario y percepción institucional de los estudiantes. En comparación, las demás propuestas desarrolladas que desarrollaron temáticas relacionadas con obstáculos y desafíos de profesores y estudiantes de Pedagogía en Educación Física (Navarro et al., 2021), perfiles de egreso de carreras de Pedagogía en Educación Física de universidades (Flores et al., 2020), creación y validación de un instrumento para valorar la calidad de la formación de profesores de Educación Física en Chile (Gonzalez et al., 2020), declaración de contenidos relacionados con AEM desde programas de estudio (Reyno, 2010) y una revisión crítica de diseños curriculares, materias, contenidos y descriptores de las universidades en la formación docente de profesores de Educación Física desde los principios y fundamentos de la Praxiología Motriz (Jiménez, Vergara y Moreno, 2013).

Finalmente, las investigaciones desarrolladas en contextos relacionados desde las «Políticas públicas», se evidenciaron trabajos de reflexión y revisión histórica de la Educación Física en Chile, abordando temáticas relacionadas con la historia de la Educación Física (Véliz, 2015), el currículum y la colaboración pedagógica fragmentada y globalizadora (Rannau, 2020), formación docente de Pedagogía en Educación Física (CastilloRetamal et al., 2020), análisis de documentos desde la mirada de dos autores y su influencia en la asignatura de Educación Física actual (Rivera y Henríquez-Alvear, 2019), la construcción disciplinar de la Educación Física, su relación y efecto sobre la población indígena (Rivera y Henríquez, 2018), reflexión transformadora de la Educación Física y la Salud (López, 2016), implementación y desarrollo de la Educación Física en Chile desde la política y actores propulsores de corrientes particulares (Acuña, 2020; Martínez, 2011; Martínez, 2012; Riobó y Villarroel, 2019), análisis de documentos ministeriales y programas de enseñanza básica y media (Moreno, Gamboa y Poblete, 2014) y análisis, propuestas y consideraciones reflexivas de la evaluación SIMCE de Educación Física (Curilem, 2017; RodríguezRodríguez, 2016). 


\section{Conclusión}

De las 31 investigaciones seleccionadas, presentan análisis de la historia, aplicaciones prácticas y documentos de reflexión. En sus intervenciones con escolares, docentes o estudiantes de pedagogía en Educación Física nos dan a conocer la actual situación de la normativa chilena sobre la Educación Física, la reflexión docente en relación con el sentir de la pedagogía y la dualidad de la simplicidad de la disciplina, la complejidad de llevarlas al papel al momento de planificar y llevarlas a cabo al momento del ejercicio de la profesión. Además, nos invitan a reflexionar y dar un contexto histórico del génesis de la pedagogía en educación física en nuestro país.

La presente revisión permitió trazar preferencias de investigación desde la academia, relacionadas con la Pedagogía en Educación Física, su instalación en Chile se inició como «Pedagogía preventiva» contra la mortandad infantil, a lo largo de la historia y a través de las escuelas y corrientes en el mundo, nos ha llevado a esclarecer que la disciplina propiamente tal debe dar respuesta a la tridimensionalidad del ser humano, desde la perspectiva biológica, psicológica y social, sin dejar de lado que el génesis de la «Educación Física» ha estado ligada a la supervivencia del ser humano como especie.

\section{Aplicaciones prácticas en base a la revisión}

Se sugiere la implementación de manera progresiva de corrientes o escuelas de la Educación Física en niños, niñas y adolescentes en contextos escolares y desde la formación inicial docente desde la Universidad. La implementación de manera progresiva permitirá sustentar el desarrollo biopsicosocial de los educandos, entregando herramientas ligadas a las perspectivas biológica, psicológica y social que posee el ser humano, permitiendo el desarrollo de un ser integro para la sociedad. Las actividades deben ir desde lo más sencillo hasta lo más complejo a través de intervenciones de actividades físico - deportivas o juegos grupales, sin dejar de lado aspectos socioemocionales que fortalecen vínculos de trabajo colaborativo y el disfrute por la práctica de la Educación Física.

\section{Futuras líneas de investigación}

Este trabajo, permitió concluir el contexto histórico y actual de la Educación Física en Chile, sus implicancias en la realidad educativa y social a través del desarrollo de las investigaciones de pregrado y postgrado.

Sin embargo, a pesar de las distintas corrientes y escuelas influyentes en la Educación Física y debido a la heterogeneidad de los programas o mallas de las Universidades, no se podría evidenciar la entrega de herramientas ligadas a las perspectivas biológica, psicológica y social que posee el ser humano en los estudiantes de Pedagogía en Educación Física y sugeridas por los autores evidenciados en esta investigación, debido que los resultados dados a conocer en las investigaciones consultadas nos entregaron distintas realidades y apreciaciones de un tema en particular. Esto, podría permitir orientar al desarrollo de actividades físico-deportivas o juegos a través de los cursos por niveles escolares o educativos propuestos por el MINEDUC, dado que en la actualidad solo se evidencia la preocupación por el bienestar físico, olvidándose de aspectos psicológicos que van de la mano con el bienestar biopsicosocial.

\section{Agradecimientos}

Agradecemos a los revisores de la presente investigación, dado a que su visión crítica y global de la experticia de esta temática, nos permitió fortalecer la investigación.

\section{Referencias}

Acuña Rojas, P. (2020). ¡ Formemos espartanos chilenos! Políticas y campañas deportivas durante la dictadura de Carlos Ibáñez, 1927-1931. Cuadernos de historia (Santiago), (52), 233-261. Doi: http://dx.doi.org/10.4067/S071912432020000100233

BND (2018). Biblioteca Nacional de Chile. Educar la fuerza y la voluntad. La Educación Física en Chile (1889-1930). Recuperado en: http://www.memoriachilena.gob.cl/602/w3article-100666.html\#presentacion

Castillo-Retamal,F.,Almonacid-Fierro,A., Castillo-Retamal,M., \& Bássoli de Oliveira,A. (2020). Formación de profesores de Educación Física en Chile: una mirada histórica (Physical Education teacher training in Chile: a historical view). Retos, 38, 317-324. Doi: https://doi.org/10.47197/ retos.v38i38.73304

Celis-Morales, C., Salas, C., Álvarez, C., Aguilar Farías, N., Ramírez Campillos, R., Leppe, J., ... \& Martínez, M. A. (2015). Un mayor nivel de actividad física se asocia a una menor prevalencia de factores de riesgo cardiovascular en Chile: resultados de la Encuesta Nacional de Salud 2009-2010. Revista médica de Chile, 143(11), 1435-1443.

Curilem, C. A. (2017). Evaluación y diseño de un Sistema de medición de la calidad de la Educación fisica (Doctoral dissertation, Universidad de Córdoba). https://dialnet.unirioja.es/servlet/ tesis? codigo $=70564$

Díaz-Martínez, X., Garrido, A., Martínez, M. A., Leiva, A. M., 
Álvarez, C., Ramírez-Campillo, R., ... \&Abraña,A. M. (2017). Factores asociados a inactividad física en Chile: resultados de la Encuesta Nacional de Salud 2009-2010. Revista médica de Chile, 145(10), 1259-1267.

Flores Ferro, E., Escobar Ruiz, N. , Jara Rojas, P., Maureira Cid, F., Gutiérrez Duarte, S., Cárdenas Begazo, S., MuñozLara, M., \& Díaz Magallanes, V. (2020a). Análisis del perfil de egreso de la carrera de pedagogía en educación física de Chile: un estudio cuantitativo (Analysis of the graduate profile of Chile's physical education pedagogy degree: a quantitative study). Retos, 39, 532-538. Doi: https://doi.org/10.47197/ retos.v0i39.81379

Flores Ferro, E., Maureira Cid, F., Silva Salse, Ángela, Muñoz Lara, M., Matheu Pérez, A., Navarro Aburto, B., Bahamondes Acevedo, V., Hadweh Briceño, M., Hurtado Almonacid, J., Valdés Montecinos, M., Aedo Muñoz, E., PeñaTroncoso, S., SereyAraneda, D. , Zavala Crichton, J. P., Maureira Sánchez, J., BrevisYeber, M., \& Valero Catalán, M. (2020b). Percepciones institucionales de los estudiantes de Pedagogía en Educación Física de Chile (Institutional perceptions of Physical Education Pedagogy students from Chile). Retos, 38, 312-316. Doi:https:/ /doi.org/10.47197/retos.v38i38.76710

Flores Ferro, E., Maurera Cid, F., Hadweh Briceño, M., Alonso Gutiérrez Duarte, S., Silva-Salse, Ángela, Peña-Troncoso, S., Castillo-Retamal, F., González Flores, P., Pauvif Cárcamo, F., Bahamondes Acevedo, V., ZapataVera, G., Zavala-Crichton, J. P., Maureira Sánchez, J., Brevis-Yever, M., \& Lagos Olivos, C. (2020c). Nivel de satisfacción de las clases online por parte de los estudiantes de Educación Física de Chile en tiempos de pandemia (Level of satisfaction of online classes by students of Physical Education of Chile in times of pandemic). Retos, 41, 123-130. Doi: https://doi.org/10.47197/ retos.v0i41.82907

Flores, E., Bahamondes, V.V., Maureira, F., \& González, P. (2019). Motivos de deserción universitaria de estudiantes de educación física de Chile. EmásF: revista digital de educación física, (57), 14-23. https://dialnet.unirioja.es/servlet/ articulo? codigo $=6860150$

Franco, E. (2010). Evolución diacrónica del concepto de Educación Física. Revista Digital EF Deportes, 15, 147. Recuperado en: https: / / www.efdeportes.com/efd147/evoluciondiacronica-del-concepto-de-educacion-fisica.htm

Galvez C., Espinoza E., Veliz C., Flores Ferro E., \& Maureira F. (2015). Factores motivacionales en la práctica de actividad física de los estudiantes de Educación Física de Santiago de Chile. VIREF Revista De Educación Física, 4(3), 74-86. Recuperado a partir de https://revistas.udea.edu.co/index.php/ viref/article/view/23172

García,A., \& Ruiz, J. A. (2010). La Educación Física en la historia del mundo contemporáneo. Lecturas, Educación Física y deportes, 148. Recuperado en:https: / www.efdeportes.com/ efd148/la-educacion-fisica-en-la-historia.htm

Giakoni, F., Paredes, P., \& Duclos-Bastías, D. (2021). Educación Física en Chile: tiempo de dedicación y su influencia en la condición física, composición corporal y nivel de actividad física en escolares. Retos, 39, 24-29. Doi: https://doi.org/ 10.47197/retos.v0i39.77781

Gonzalez Orb, M., Molina Sotomayor, E., Ferreira Urzúa, M., Leiva Contardo, R., Martínez Salazar, C., Trujillo Galindo, H., Lobos González, M., Beltran Gonzalez,A., VargasVitoria, R., FlandezValderrama, J., \& Negron Molina, M. (2019). Construcción y validación de un instrumento para valorar la calidad de la formación de profesores de Educación Física en Chile (Construction and validation of an instrument for assessing training quality in physical education teachers from Chile). Retos, 37, 312-319. Doi: https://doi.org/10.47197/ retos.v37i37.69304

González, R., Madrera, E., \& Salguero, A. (2004). Las escuelas gimnásticas y su relación con la actividad física y educación física actuales. Revista Digital EF Deportes, 10, 73. Recuperado en: https: / www.efdeportes.com/efd73/gimn.htm

Jiménez, G., Vergara, M., \& Moreno, J.H. (2013). Análisis pedagógico de los contenidos curriculares de la formación de los docentes de Educación Física en Chile a la luz de la Praxiología Motriz. Revista Iberoamericana de Educación, 61(4), 6. https: / / dialnet.unirioja.es $/$ servlet $/$ articulo?codigo $=5891888$

JUNAEB (2017). Informe Mapa Nutricional 2017. Disponible en: https://www.junaeb.cl/wp-content/uploads/2013/ 03/Informe-Mapa-Nutricional-2017-FINAL.pdf

López, A. A. (2016). Buen vivir, sistema escolar y educación física en chile: una realidad por construir. Revista Infancia, Educación y Aprendizaje, 2(2), 138-149. https://panambi.uv.cl/ index.php/IEYA/article/view/599

Luarte R., C., Poblete V., F., \& Flores R., C. (2014). Nivel de desarrollo motor grueso en preescolares sin intervención de profesores de educación física, Concepción, Chile. Ciencias De La Actividad Física UCM, 15(1), 7-16. Recuperado a partir de http:/ / revistacaf.ucm.cl/article/view/36

Mamonde, M. (1995). Educación física militarizada en Argentina. Educación Física y Ciencia, Facultad de Humanidades y Ciencias de la Educación, Universidad Nacional de La Plata. 1. Recuperado en: http://sedici.unlp.edu.ar/handle/10915/ 11742

Martínez, F. (2011). Construyendo cuerpos infantiles... modelando cuerpos patriotas. La educación física en chile. 1889-1920. Educación Física y Ciencia, 13, 99-10. http:// w w w. me moria.fah ce . un l p.edu . a r / library $?_{\mathrm{a}}=\mathrm{d} \& \mathrm{c}=\operatorname{arti} \& \mathrm{~d}=\operatorname{Jpr} 5229$

Martínez, F. (2012). Hacia una pedagogía del cuerpo: la Educación Física en Chile. 1889-1920. Ágora para la Educación Física y el Deporte, 14(3), 320-331.https: / / dialnet.unirioja.es/servlet/ articulo?codigo $=4103298$

Maureira Cid,F.,Flores Ferro, E., GonzálezFlores, P., Palma Gajardo, E., Fernández Rebolledo, M., \& Véliz Véliz, C. (2018). Propiedades psicométricas del inventario de estilos de aprendizaje de Kolb y del cuestionario de Felder-Silverman en estudiantes de educación física de Santiago de Chile. Ciencias De La Actividad Física UCM, 19(1), 1-11. Doi: https://doi.org/ 10.29035/rcaf.19.1.5

Maureira, F. (2013). Validez y confiabilidad del CHAEA en estu- 
diantes de educación física de Chile. Educación física Chile, (271), 3. https://dialnet.unirioja.es/servlet/ articulo?codigo $=7692455$

Maureira, F. (2021). Conocimientos biológicos de la homosexualidad y transexualidad en estudiantes de Educación Física de Chile (Biological knowledge of homosexuality and transsexuality in Physical Education students in Chile). Retos, 42, 805-812. Doi: https://doi.org/10.47197/ retos.v42i0.88708

Maureira, F., Flores, E., Castillo, F., Cortés, M. C., Peña, S., Bahamonde, V.., ... \& Cortes, B. (2021b)Prevalencia de neuromitos en estudiantes de Pedagogía en Educación Física de Chile (Prevalence of neuromyths in students of Physical Education Pedagogy of Chile). Retos, 42,426-433. Doi: https: / /doi.org/10.47197/retos.v42i0.88204

Maureira, F., Flores, E., Ramírez, M.A., Cortes, B., \& Hernández, P. B. (2021a). Relación de los estilos de aprendizaje, habilidad emocional, habilidades múltiples y detección emocional en estudiantes de educación física de Santiago de Chile. Ciencias de la Actividad Física UCM, 22(2), 1-13. Doi: https://doi.org/ 10.29035/rcaf.22.2.1

Moreno Doña, A., Campos Vidal, M., \& Almonacid Fierro, A. (2012). Las funciones de la educación física escolar: una mirada centrada en la justicia social y la reconstrucción del conocimiento. Estudios pedagógicos (Valdivia), 38(ESPECIAL), 1326. http://dx.doi.org/10.4067/S0718 07052012000400002

Moreno, A., Gamboa, R., \& Poblete, C. (2014). La Educación Física en Chile: análisis crítico de la documentación ministerial1. Revista Brasileira de Ciências do Esporte, 36, 411-427. Doi: https: / /doi.org/10.1590/S0101-32892014000200010

NavarroAburto, B., PeñaTrocoso, S., Beltrán, J.C., Guzmán Muñoz, A., Flores Ferro, E., \& Fuentes Merino, P. (2021). Desafíos de las Estrategias Pedagógicas Integradas (EPI) en la formación de profesores y profesoras de Educación Física en Chile (Challenges of integrated learning Strategies (ILS) in a Physical Education teaching training program in Chile). Retos, 42, 750756. Doi: https://doi.org/10.47197/retos.v42i0.80079

Petermann, F., Durán, E., Labraña,A.M., Martínez, M.A., Leiva,A. M., Garrido-Méndez,A., ... \& Celis-Morales, C. (2017). Factores asociados al desarrollo de obesidad en Chile: resultados de la Encuesta Nacional de Salud 2009-2010. Revista médica de Chile, 145(6), 716-722

Poblete, C., Moreno,A., \& Rivera, E. (2014). Educación Física en Chile: Una historia de la disciplina en los escritos de la primera publicación oficial del Instituto de Educación Física de la Universidad de Chile (1934-1962). Estudios pedagógicos (Valdivia), 40(2), 265-282. Recuperado en: https: / / scielo.conicyt.dl/scielo.php?script $=$ sci_arttext\&pid $=$ S071807052014000300016

RAE (2019). Diccionario de la lengua española. Edición del Tricentenario. Educación Física. Recuperado en: https:// dle.rae.es/educaci\%C3\%B3n\#1YqnuKu

Ramos, A., López, R., \& Murillo, J. (2017). Alfabetización física: una percepción reflexiva. Dialógica: revista multidisciplinaria, 14(1), 87-102. https://dialnet.unirioja.es/ servlet/articulo?codigo $=6216222$

Rannau, J. P. (2020). Educación Física en Chile: hacia una transdiciplinariedad desde elcurrículum y la colaboración pedagógica. Praxis Educativa, 24 (2), 1-17. Doi: https:// dx.doi.org/10.19137/praxiseducativa-2020-240210

Reyno,A. (2010a). Contenidos de la actividad expresivo motriz en los programas de las carreras de pedagogía en Educación Física, en Chile.(Content Related to Body Expression Activities inTeaching Degree Programs in Physical Education, in Chile). Cultura, Ciencia y Deporte, 5(13), 5-10. Doi: https:/ / doi.org/ 10.12800/ccd.v5i13.61

Reyno, A. (2010b). Los objetivos y contenidos de las actividades de expresión motriz, según la opinión de los profesores de educación física en Chile (Objectives and contents of the activities of motor expression, according to the opinion of Physical Education teachers in C. Retos, 18, 56-59. https: / / doi.org/10.47197/retos.v0i18.34653

Reyno, A. (2011). Los contenidos de la expresión motriz y la gimnasia rítmica según la opinión de los profesores de Educación Física en Chile. EmásF: revista digital de educación física, (12), 9-21. https://dialnet.unirioja.es/servlet/ articulo?codigo $=3890821$

Riobó, E., \& Villarroel, F. J. (2019). Belleza plástica, eugenesia y educación física en Chile: presentación de la fuente «Aspectos de la educación física», de Luis Bisquertt (1930). História, Ciências, Saúde-Manguinhos, 26, 673-682. Doi: https:// doi.org/10.1590/S0104-59702019000200018

Rivera Gutiérrez, C., \& Henríquez-Alvear, L. (2019). Análisis de los documentos (1872-1886) que influyeron en los programas de la disciplina de Educación Física en Chile. Journal of Sport \& Health Research, 11 (2). https: / / recyt.fecyt.es/ index.php/JSHR/article/view/80953

Rivera, C., \& Henríquez, L. 2018). Construcción disciplinar de la educación física, efectos sobre una región culturalmente diversa: La Araucanía. Revista Inclusiones, 174-194. http:// revistainclusiones.org/index.php/inclu/article/view/918

Rodríguez-Rodríguez, F. (2016). Medición de la calidad de la Educación Física en Chile, un desafío pendiente. Espacios en Blanco. Revista de Educación (Serie Indagaciones), 26 (2). h t t p : / / w w w. s c i e lo.org.a r / scielo.php?script $=$ sci_arttext\&pid $=S 1515$ 94852016000200002\&lang $=\mathrm{es}$

Sainz, R. (1992). Historia de la educación física. Cuadernos de sección. Educación, 5, 27-47. https://www.euskoikaskuntza.eus/es/publicaciones/historia-de-laeducacion-fisica/art-11632/

Veliz Veliz C., Arenas San Martín R., Gálvez Mella C., Bahamondes AcevedoV., \& Maureira Cid F. (2015). Breve historia de la Educación Física en Chile. VIREF Revista De Educación Física, 4(2), 99-110. Recuperado a partir de https://revistas.udea.edu.co/index.php/viref/article/ view/23656

Zagalaz, M., Moreno, R., Cachón, J. (2001). Nuevas tendencias en la Educación Física. Contextos Educativos, 4, 263 294. Recuperado en: https: / / publicaciones.unirioja.es/ ojs/index.php/contextos/article/view/497/461

$-915-$ 